\title{
Libre Software in Spanish Public Administrations
}

\author{
Felipe Ortega, Isabel Lafuente, Jose Gato, and Jesús M. González-Barahona \\ GSyC/LibreSoft \\ $\{j f e l i p e$, islafuente, jgato, jgb\} @gsyc.es
}

Libre software started to be used in Public Administrations in Spain during the 1990s, in some isolated but interesting experiences. During the early 2000s, and specially in some regional governments, libre software started to be considered as an integral part of ITrelated policies. In 2007, it was evident that many experiences related to libre software were running in Public Administrations with different levels of success. However, no study had looked into the details of these experiences, and no comprehensive analysis had been performed to better understand the different factors that affect them.

From September 2007 to May 2008 the GSyC/LibreSoft group of the Universidad Rey Juan Carlos and Telefonica I+D studied the situation of libre (free, open source) software in Public Administrations in Spain. The study was funded by CENATIC, a public Foundation interested in the promotion of libre software in Spain, which also participated in its elaboration. The aim was to document in some detail several of the most interesting experiences, and obtain some insight about how libre software can be used in the context of Public Administrations.

The methodology used to conduct the research was based primarily on the critical analysis of reports already available, in-depth interviews with relevant experts in the area, and further elaboration of this material, with several phases of feedback. As a result, a repor 11 with the main results was presented in Madrid in October 2008. Many of its conclusions are relevant not only to the Spanish context, but can be extended to many other regions where Public Administrations are experimenting with libre software.

${ }_{1}^{1}$ http://observatorio.cenatic.es/infcenatic01.pdf

C. Boldyreff et al. (Eds.): OSS 2009, IFIP AICT 299, p. 366, 2009.

(C) IFIP International Federation for Information Processing 2009 